\title{
Review Article \\ The Effect of Hepatosteatosis on Response to Antiviral Treatment in Patients with Chronic Hepatitis B: A Meta-Analysis
}

\author{
Yongfen Zhu, Qiao Yang, Fangfang Lv, and Yunsong Yu \\ Department of Hepatology and Infection, Sir Run Run Shaw Hospital, Zhejiang University, Hangzhou, China \\ Correspondence should be addressed to Yongfen Zhu; yongfen1978@126.com
}

Received 17 December 2016; Accepted 8 February 2017; Published 21 March 2017

Academic Editor: Paul Enck

Copyright (c) 2017 Yongfen Zhu et al. This is an open access article distributed under the Creative Commons Attribution License, which permits unrestricted use, distribution, and reproduction in any medium, provided the original work is properly cited.

\begin{abstract}
Background. This study is to systematically analyze the effects of hepatosteatosis on the response to antiviral treatment in patients with chronic hepatitis B (CHB) and hepatosteatosis. Methods. Systematic search was performed in PubMed, Embase, Web of Science, Elsevier, and the Chinese BioMedical literature databases for relevant studies published until February 2016. Treatment outcomes were compared between patients with CHB plus concomitant hepatosteatosis and those without hepatosteatosis. Results. A total of 8 prospective cohort studies (399 patients with CHB plus hepatosteatosis and 688 patients with only $\mathrm{CHB}$ ) were included. Biochemical and virological response at both 48 and 96 weeks were significantly lower in patients with $\mathrm{CHB}$ plus hepatosteatosis as compared to that in patients with only CHB. Subgroup analysis based on methods used for diagnosis of hepatosteatosis and treatment regimens showed that when hepatosteatosis was diagnosed on Doppler ultrasound and treated with nucleotide analogues, patients with $\mathrm{CHB}$ plus hepatosteatosis showed lower biochemical $(62.7 \%$ versus $75.8 \%, P=0.002)$ and virological response $(66.2 \%$ versus $72.3 \%$, $P=0.006)$ as compared to that in patients with CHB. Conclusion. Hepatosteatosis lowers the efficacy of antiviral treatment in patients with $\mathrm{CHB}$, especially when hepatosteatosis was diagnosed on ultrasound findings and treated with nucleotide analogues.
\end{abstract}

\section{Introduction}

Hepatitis B virus (HBV) infection is one of the main causes of chronic liver disease and accounts for more than 350 million people with chronic hepatitis B (CHB) worldwide. Patients with persistent $\mathrm{HBV}$ infection show wide variability in clinicopathological manifestations ranging from minimal histological changes to liver cirrhosis, hepatocellular carcinoma (HCC), or even acute-on-chronic liver failure $[1,2]$. Nonalcoholic fatty liver disease (NAFLD) is characterized by fat deposition in hepatocytes and is associated with liver damage ranging from simple steatosis to liver fibrosis, cirrhosis, and HCC [3]. With socioeconomic development and lifestyle changes, NAFLD is increasingly being recognized as a public health concern with estimates of prevalence ranging from 5 to $40 \%$ in the general population [4]. It is now becoming the most common liver disease in the general population worldwide [5].

An increase in patients who have $\mathrm{CHB}$ with concomitant NAFLD has been reported [6,7]. Since the pathogenesis of CHB and NAFLD is complex, they may affect each other. Thus, coexistence of CHB and NAFLD may exhibit novel pathophysiological characteristics.

Early stage of NAFLD is defined as the presence of steatosis in more than $5 \%$ of hepatocytes [8]. HBV X protein is reported to induce hepatic steatosis by enhancing the expression of liver fatty acid binding proteins [9]. Recent studies have revealed a relatively common finding of steatosis in $\mathrm{CHB}$ patients; further, the incidence of steatosis is much higher in patients with $\mathrm{CHB}$ as compared to that in the general population, implying its role in $\mathrm{CHB}$ [8] Moreover, both HBV infection and steatosis can lead to necroinflammation 
in the liver. Thus, it is difficult to distinguish the cause of hepatic necroinflammation. Therefore, the presence of hepatic steatosis may adversely affect the efficacy of antiviral therapy $[10,11]$.

Several recent clinical studies have investigated the impact of superimposed hepatosteatosis on the response to antiviral treatment in patients with $\mathrm{CHB}$; however, the results have been inconsistent. We conducted a meta-analysis to systematically analyze the effects of hepatosteatosis on the response to antiviral treatment in patients with $\mathrm{CHB}$ and hepatosteatosis.

\section{Methods}

2.1. Search Strategy and Study Selection. Systematic search was performed on PubMed, Embase, Web of Science, Elsevier, and the Chinese BioMedical literature databases for articles published as of February 2016. The following keywords were used during the search: "chronic hepatitis B" or "inflammation of liver caused by hepatitis B virus"; "hepatic steatosis" or "hepatosteatosis" or "fatty liver" or "NAFLD" or "NASH"; and "antiviral therapy" or "nucleotide analogue" or "peginterferon alfa" or "standard interferon alfa." Titles and abstracts of retrieved studies were first scanned, and the full texts of potential eligible studies were reviewed. The retrieved studies were carefully examined to exclude potential duplicates or papers with overlapping data. Only full-text publications compared the response to antiviral treatment in patients with $\mathrm{CHB}$ and concomitant hepatosteatosis with those in $\mathrm{CHB}$ without hepatosteatosis. Studies that were not published as full reports, such as conference abstracts and letters to the editors, were excluded. Reports cited in the references and relevant reviews were also manually searched to include potentially missed studies.

2.2. Data Extraction and Outcome Definitions. Data was extracted independently by two authors; any discrepancies were resolved by consensus amongst the authors. The following information was extracted from each trial: publication details (title, the first author, and place of the study conducted), study design (inclusion and exclusion criteria), participant details (the numbers of patients enrolled, age), intervention details (including type and dose of interferon, nucleotide analogue, and mode of administration), duration of treatment and follow-up, and outcomes. The outcomes included biochemical response (time taken for the serum levels of aminotransferase to return to normal), virological response (time taken for the HBV DNA to become undetectable), and serological response (time taken for the disappearance of $\mathrm{HBeAg}$ and the appearance of anti-HBe).

Quality assessment of the included studies was done by two authors using an improved Newcastle-Ottawa Scale [12]. Studies which scored $\geq 9$ points were deemed to be of high quality; those with $5-8$ points and $<5$ points were deemed to be of moderate and low quality, respectively.

2.3. Statistical Analysis. Heterogeneity between individual studies was assessed by $I^{2}$ test. A value greater than $75 \%$ was considered indicative of a substantial heterogeneity; that between $50 \%$ and $75 \%$ was considered indicative of moderate heterogeneity; that between $25 \%$ and $50 \%$ was considered indicative of mild heterogeneity; and a value $<25 \%$ was considered indicative of absence of heterogeneity. A random effects model was used in the event of significant heterogeneity; a fixed effects model was used otherwise. The impact of publication bias was assessed using the Egger regression asymmetry test. Funnel plots were constructed if a sufficient number of studies with low heterogeneity were available. A $P$ value $<0.05$ was considered statistically significant. STATA 11.0 software (Stat Corporation, College Station, Texas, USA) was used for all analyses.

\section{Results}

3.1. Search Results and the Characteristics of the Included Studies. A total of 1030 articles were retrieved on initial literature search, of which 28 were deemed to be potentially relevant on a review of titles and abstracts. After a careful review of the 28 full-text articles, 3 were excluded owing to data duplication; four were excluded due to the lack of a control group; two were excluded because of inadequate duration of antiviral treatment; 11 were excluded as the NOS scores were $<6$. Finally, a total of 8 articles were included in the meta-analysis [13-20] (Figure 1).

The general information of the included studies is shown in Table 1. Amongst these studies, 2 were conducted in Turkey $[13,16]$ and 6 in China $[14,15,17-20]$. Five studies were published in English; three were in Chinese. In four studies, patients were treated with only interferonalpha $[13,17,19,20]$; in three studies, patients were treated only with nucleoside analogues $[14,15,18]$; and in one study, the patients were treated with interferon-alpha in combination with nucleoside analogues [19]. Four trials are comprised of 48-week interferon-alpha treatment; two were with 48-week follow-up [19, 20] and the other two were with 96-week follow-up [13, 17]. One trial is comprised of 48-week treatment with interferon-alpha combined with nucleoside analogues and 48-week follow-up [16]. Three trials are comprised of 96-week treatment with nucleoside analogues and 96-week follow-up [14, 15, 18]. Four studies were prospective cohort studies [13, 17-19] while the other four studies were retrospective cohort studies [14-16, 20].

Baseline data, including alanine aminotransferase (ALT), aspartate aminotransferase (AST), HBV DNA level, and the percentage of patients who were $\mathrm{HBeAg}$ positive, are shown in Table 2.

\subsection{Biochemical, Virological, and Serological Responses at} 48 Weeks. Five trials with a combined study population of 325 patients with $\mathrm{CHB}$ plus steatosis and 530 patients with only $\mathrm{CHB}$ reported data on biochemical response at 48 weeks $[14,15,17-19]$. The result is shown in Figure 2(a). No substantial heterogeneity was observed amongst these studies $\left(I^{2}=0 \%, P=0.083\right)$, and a fixed effects model was used for the analysis. Patients with $\mathrm{CHB}$ plus steatosis showed a lower rate of biochemical 


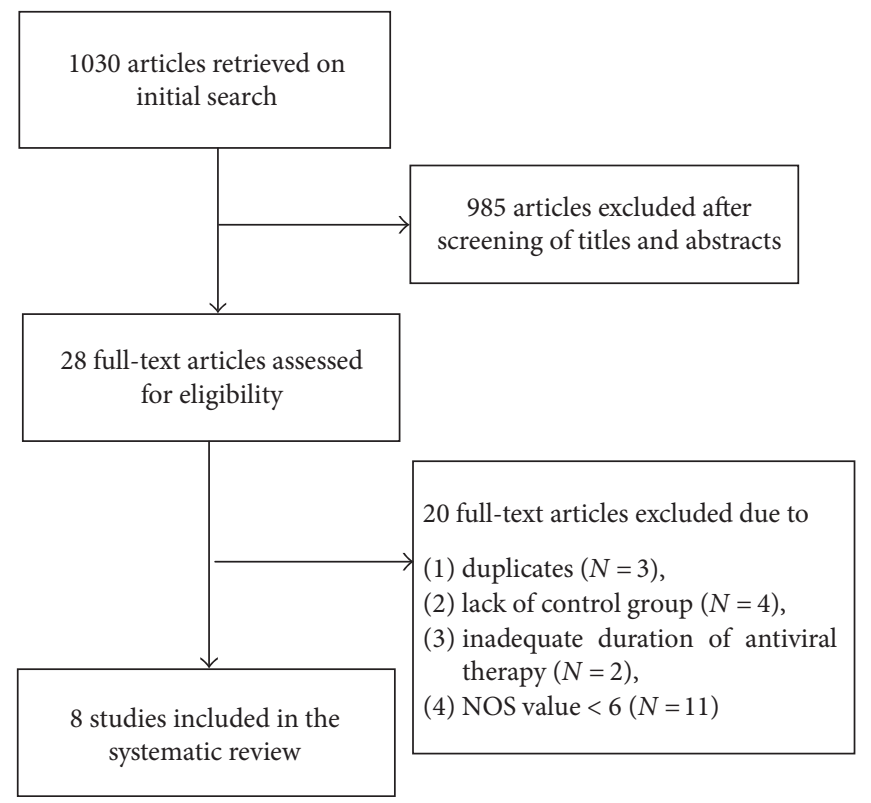

FIGURE 1: Flow chart showing study selection for the meta-analysis.

response at 48 weeks as compared to that in patients with only $\mathrm{CHB}$ (59.7\% versus $69.6 \%$; risk ratio $(\mathrm{RR})=0.86,95 \%$ CI $0.78-0.96, P=0.007)$.

Seven studies had reported data on virological response at 48 weeks $[13-16,18,19]$. No substantial heterogeneity was observed $\left(I^{2}=0 \%, P=0.95\right)$, and the fixed effects model was used. Patients with $\mathrm{CHB}$ and steatosis showed a lower rate of virological response at 48 weeks as compared to that observed in patients with only $\mathrm{CHB}$ (58.7\% versus $65.3 \%$, $\mathrm{RR}=0.90,95 \%$ CI 0.81-0.99, $P=0.03$, Figure 2(b)).

Four trials addressed the serological response to antiviral treatment at 48 weeks [17-20]. No statistically significant heterogeneity was observed amongst these studies $\left(I^{2}=0 \%\right.$, $P=0.99)$. The estimated pooled RR value showed no significant between-groups difference (27.6\% versus $29.7 \%$, $\mathrm{RR}=0.90,95 \%$ CI $0.66-1.23, P=0.504$, Figure 2(c)).

3.3. Biochemical, Virological, and Serological Responses at 96 Weeks. Five studies reported data on biochemical response at 96 weeks, which showed no heterogeneity $(P=0.178$, $\left.I^{2}=39 \%\right)[14,15,17,18]$. The pooled RR showed a significantly lower sustained biochemical response in patients with $\mathrm{CHB}$ and steatosis as compared to that in patients with only CHB (71.2\% versus $86.6 \%, \mathrm{RR}=0.85,95 \%$ CI $0.78-0.93$, $P=0.000$, Figure 3(a)).

Data on virological response at 96 weeks was available for 4 trials $[13-15,17,18]$. The pooled RR showed a significantly lower virological response in patients with $\mathrm{CHB}$ and steatosis as compared to that in patients with only $\mathrm{CHB}(67.3 \%$ versus $75.2 \%, \mathrm{RR}=0.84,95 \% \mathrm{CI} 0.78-0.92, P=0.000)$. No heterogeneity was observed amongst these studies $\left(I^{2}=46.1 \%\right.$, $P=0.12$ ) (Figure 3(b)).

Only two studies reported data on serological response at 96 weeks $[17,18]$. The fixed effects model was used for the analysis owing to no substantial heterogeneity $(P=0.51$, $\left.I^{2}=0.0 \%\right)$. No significant between-groups difference was observed with respect to sustained serological response (22.9\% versus $28.5 \%, \mathrm{RR}=0.80,95 \%$ CI $0.51-1.27, P=0.35$, Figure 3(c)).

3.4. Subgroup Analysis Based on the Method Used for the Diagnosis of Hepatosteatosis and Treatment Regimens. Next subgroup analyses on diagnosis methods and treatment regimens were performed using the outcome of virological response at 48 weeks. We found that patients diagnosed by liver biopsy were all treated with interferon, and patients diagnosed by Doppler ultrasound were all treated with nucleotide analogues. Thus, subgroup analysis was performed according to the methods used for the diagnosis of hepatosteatosis and treatment regimens: Doppler ultrasound and nucleotide analogues [14, 15, 18] or liver biopsy and interferon $[13,16,17,19,20]$. Subgroup analysis showed that if hepatosteatosis was diagnosed by liver biopsy and treated with interferon, there was no significant difference in biochemical response (47.6\% versus $48.3 \%$, $P=0.934)$, virological response $(42.5 \%$ versus $42.3 \%$, $P=0.987)$, or serological response $(34.5 \%$ versus $37.1 \%$, $P=0.718$ ). However, if hepatosteatosis was diagnosed by Doppler ultrasound and treated with nucleotide analogues, significant differences were observed in the biochemical (62.7\% versus $75.8 \%, P=0.002)$, virological $(66.2 \%$ versus $72.3 \%, P=0.006)$, and serological responses $(18.5 \%$ versus $22.3 \%, P=0.533$ ) between the two groups (Table 3, Figures 4(a), 4(b), and 4(c)).

3.5. Risk of Bias. Publication bias of the included articles was performed using Begg's and Egger's tests based on outcomes of biochemical response and virological response at 48 weeks. For biochemical response at 48 weeks, no publication bias was detected (Begg's test, $P=0.46$; Egger's test, $P=0.41$; Figure 5(a)). For virological response at 48 weeks, no 


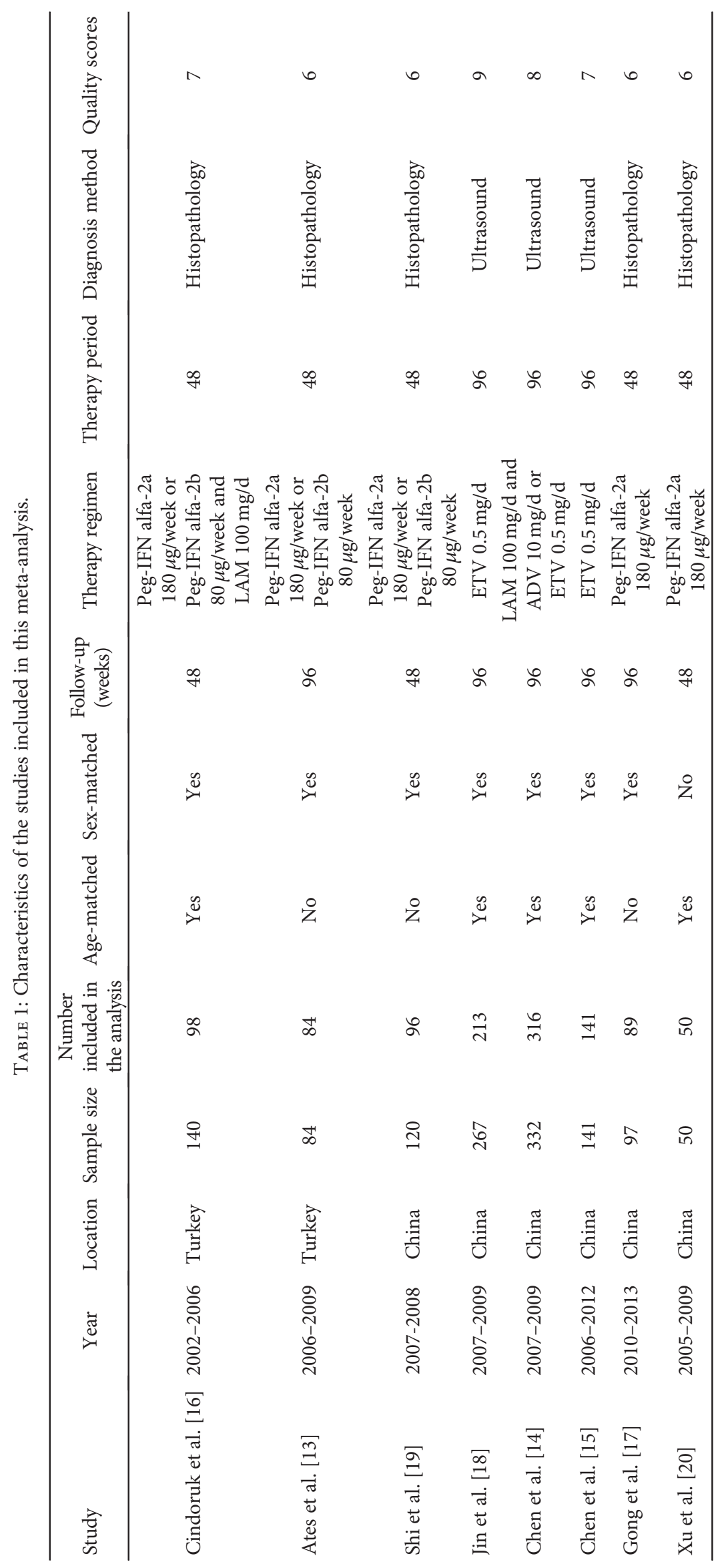




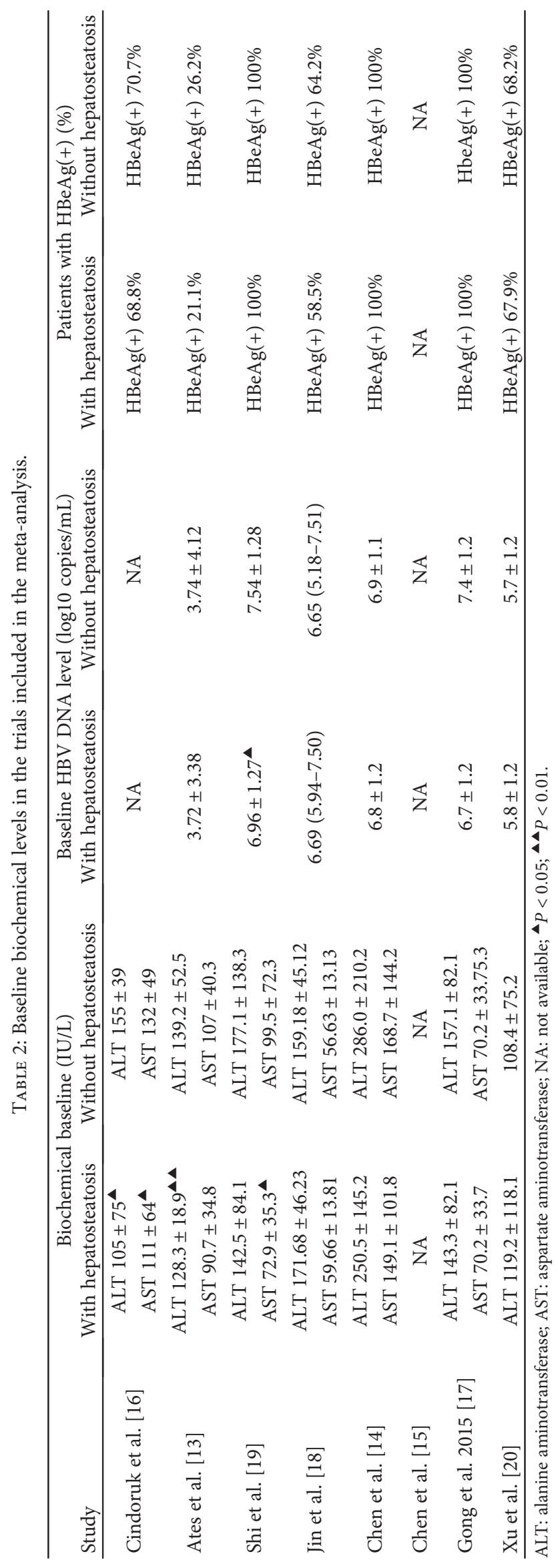




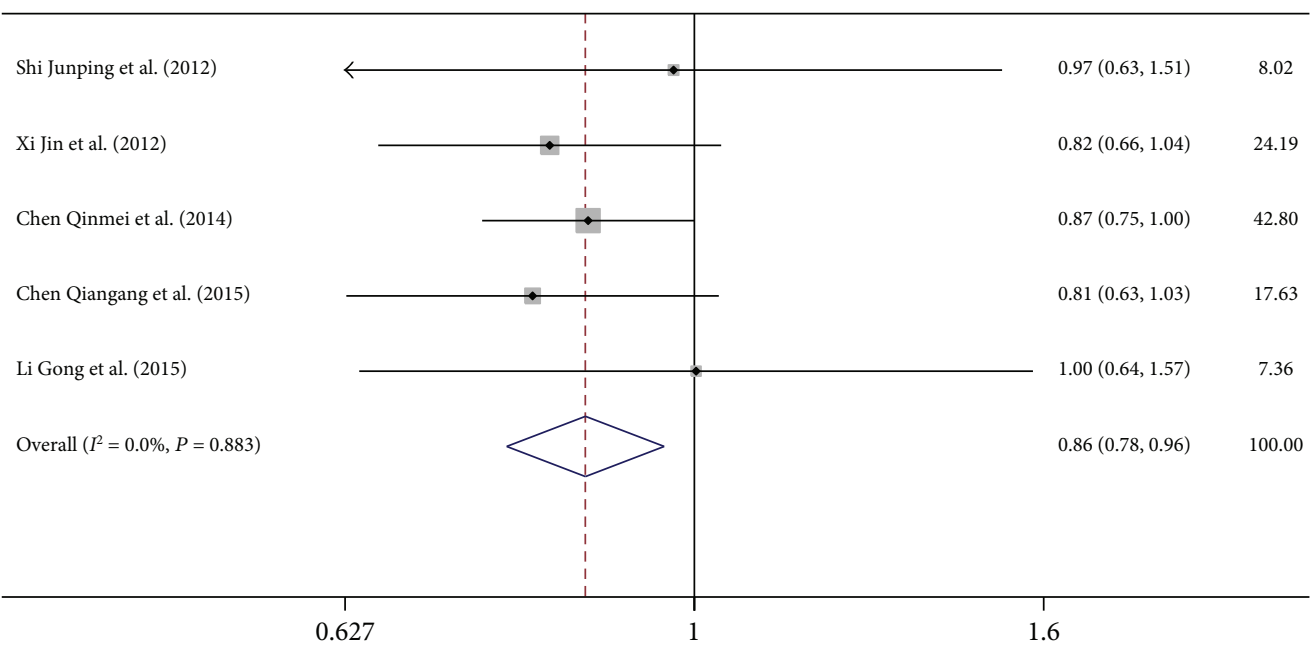

(a)

Study ID

RR (95\% CI) \% weight

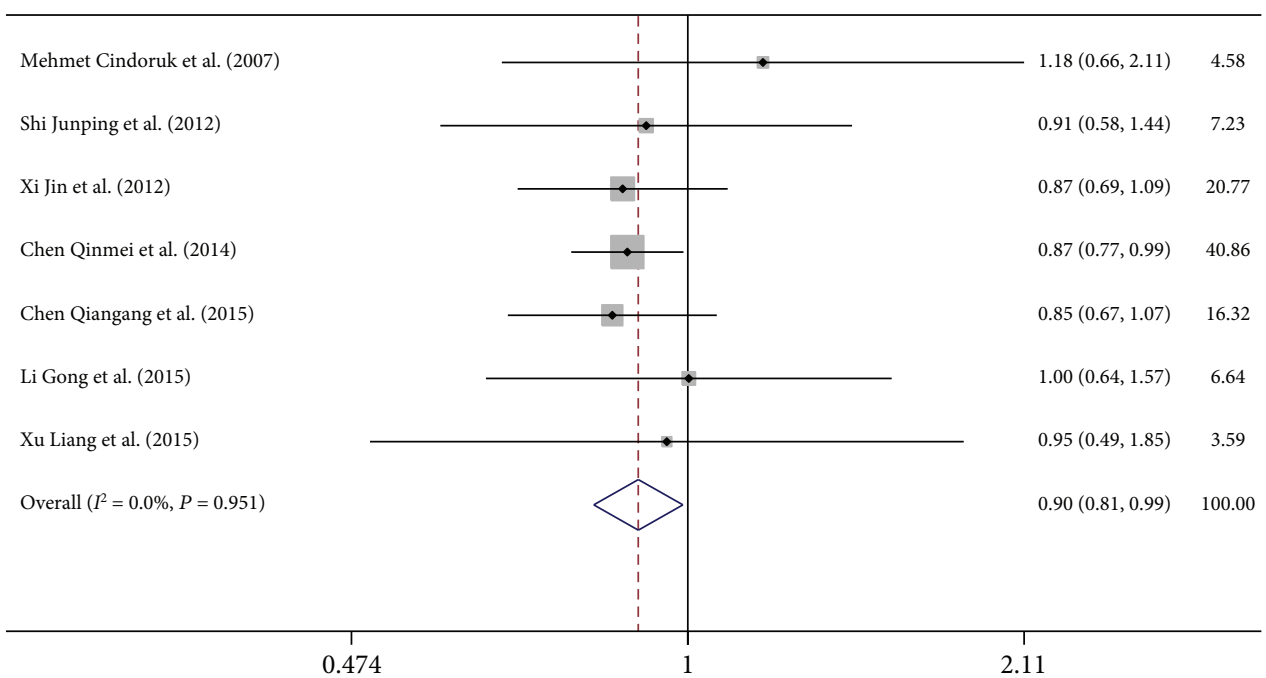

(b)

FIgure 2: Continued. 


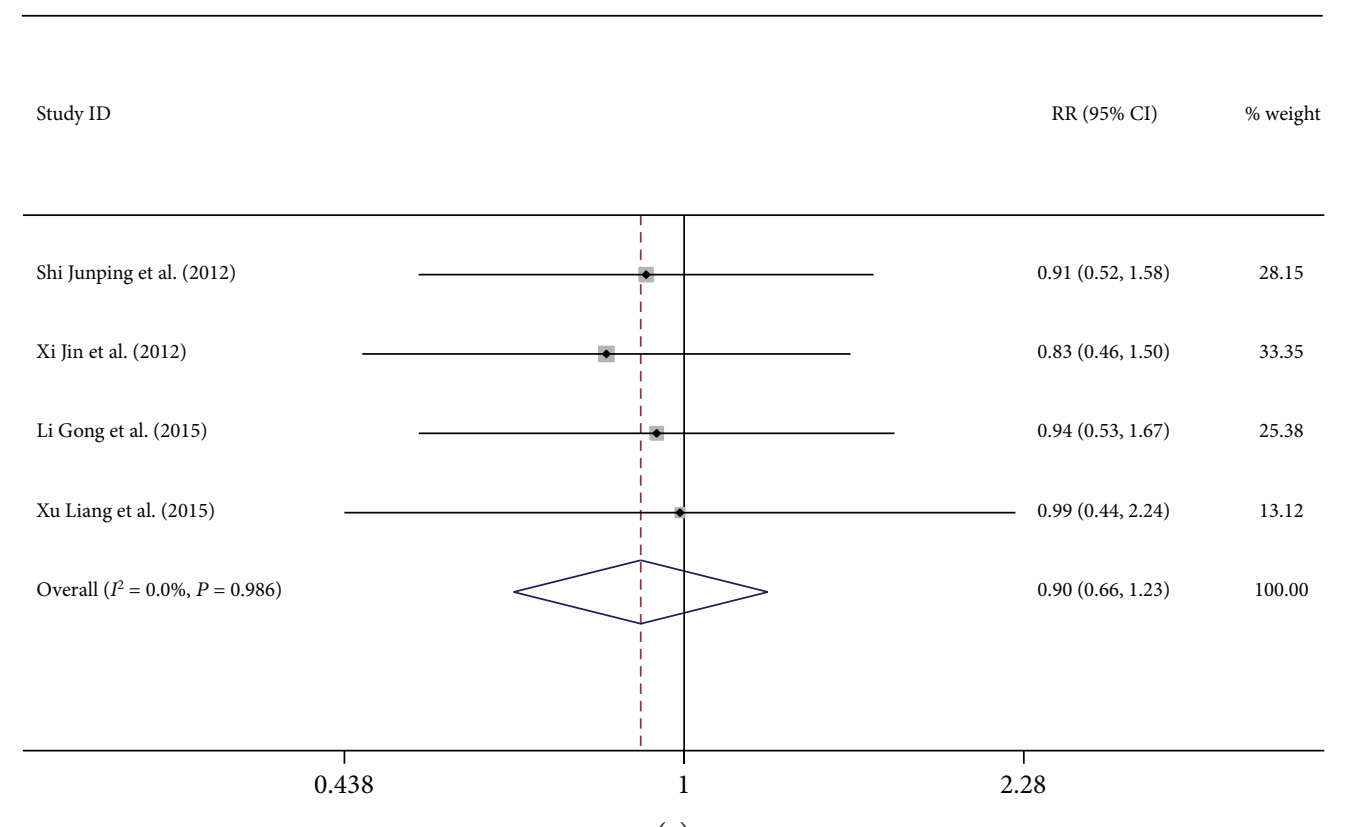

(c)

Figure 2: Forest plot of (a) biochemical, (b) virological, and (c) serological responses at 48 weeks.

publication bias was detected (Begg's test, $P=0.07$; Egger's test, $P=0.08$; Figure 5(b)).

\section{Discussion}

Due to increase in consumption of fat-rich diet coupled with sedentary lifestyle, the incidence of NAFLD is increasing [8]. Thus, the frequency of patients with $\mathrm{CHB}$ and concomitant NAFLD is also increasing. Definitive evidence of the effect of hepatosteatosis on the efficacy of antiviral therapy in patients with $\mathrm{CHB}$ is yet to be achieved. In the present meta-analysis, 8 cohort studies, published between 2002 and 2013, with a combined subject population of 399 patients with $\mathrm{CHB}$ plus hepatosteatosis and 688 patients with only $\mathrm{CHB}$, were included. All patients received interferon or nucleotide analogues for $>1$ year. The meta-analysis showed significantly lower biochemical and virological responses in CHB patients with hepatosteatosis at both 48 and 96 weeks, as compared to those in patients with only $\mathrm{CHB}$. Our data suggests that hepatosteatosis decreased the response to antiviral therapy in $\mathrm{CHB}$ patients.

Response to anti-HBV therapy is dependent on a number of variables. Of these, baseline $\mathrm{HBeAg}$ status, HBV DNA level, and $\mathrm{HBV}$ genotype are the most important predictors $[21,22]$. The studies included in the present meta-analysis did not report HBV genotypes. Except for the study conducted by Shi et al. [19], no significant difference was observed in baseline HBeAg status and HBV DNA level between the two groups. Contrary to the general belief that high HBV DNA level is a predictor of poor response to antiviral treatment [23], Shi et al. [19] found that the patients with $\mathrm{CHB}$ and hepatosteatosis had lower HBV DNA levels than the patients with $\mathrm{CHB}$ alone. This indicates that baseline HBV DNA level may not contribute to the response to treatment.

Both CHB and NAFLD cause chronic inflammation in the liver, which manifests as an increase in ALT level. It is hard to differentiate the cause of these two inflammatory diseases based on clinical presentation, though the two can be differentiated by histopathological examination. $\mathrm{CHB}$ patients show different degree of inflammatory in the portal area and surroundings, and inflammatory cells aggregate in the portal area to enlarge it; patients with NAFLD show lobular inflammatory and hepatosteatosis with low inflammatory infiltrate in the portal area, and the lobular inflammatory cell infiltration positively correlates with liver damage [24, 25]. Confirming the location of liver inflammation on biopsy can differentiate the cause of chronic inflammation.

Further subgroup analysis was performed based on the diagnostic methods and treatment regimens. It is interesting that in the included papers, when the patients were diagnosed by Doppler ultrasound, they were treated with nucleotide analogue; while when they were diagnosed by liver biopsy, they were treated with interferon. Our data showed that when hepatosteatosis was diagnosed by Doppler ultrasound and treated with nucleotide analogues, patients with $\mathrm{CHB}$ and hepatosteatosis showed lower biochemical and virological responses compared to patients with only $\mathrm{CHB}$. Such a difference was not noted between the two groups when the diagnosis of hepatosteatosis was based on biopsy and treated with interferon.

Although Doppler ultrasound has some limitations such as low sensitivity and high subjectivity in imaging diagnosis of hepatosteatosis, it has been widely applied in clinic because of noninvasion, low expense, and easy access especially in China [26]. In all the included papers, the patients with 
Study ID

RR $(95 \% \mathrm{CI})$

$\%$ weight

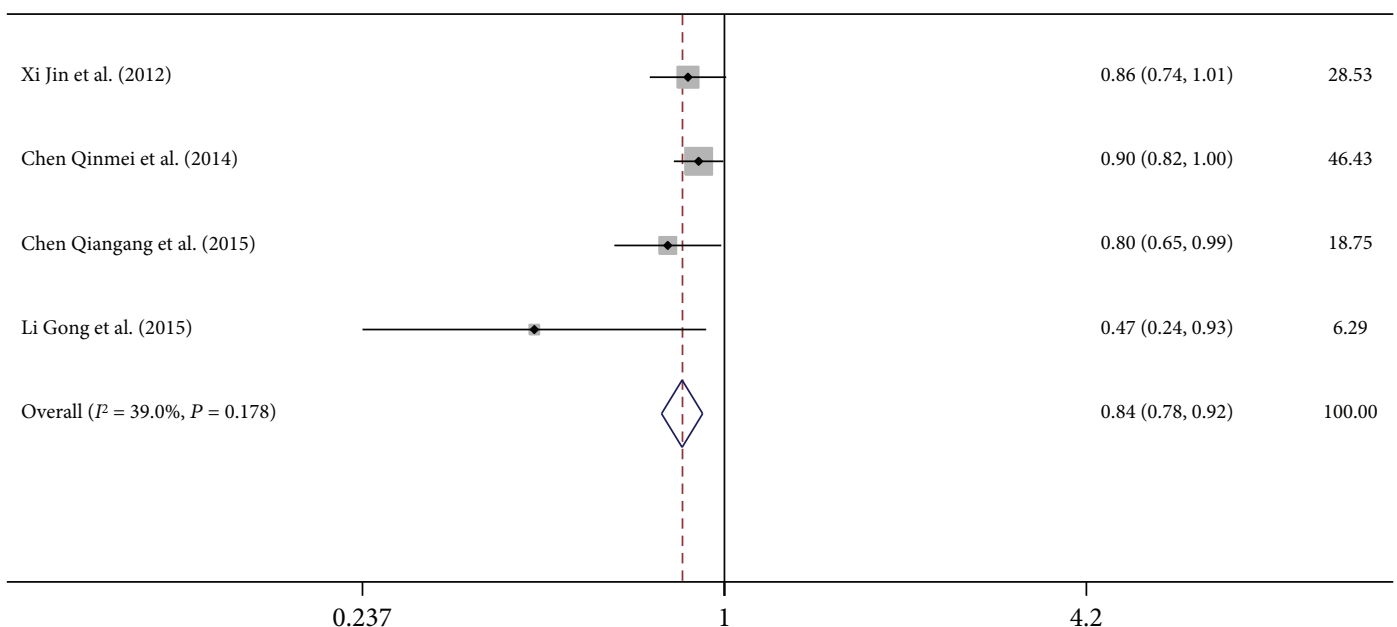

(a)

Study ID

RR $(95 \%$ CI $) \quad$ \% weight

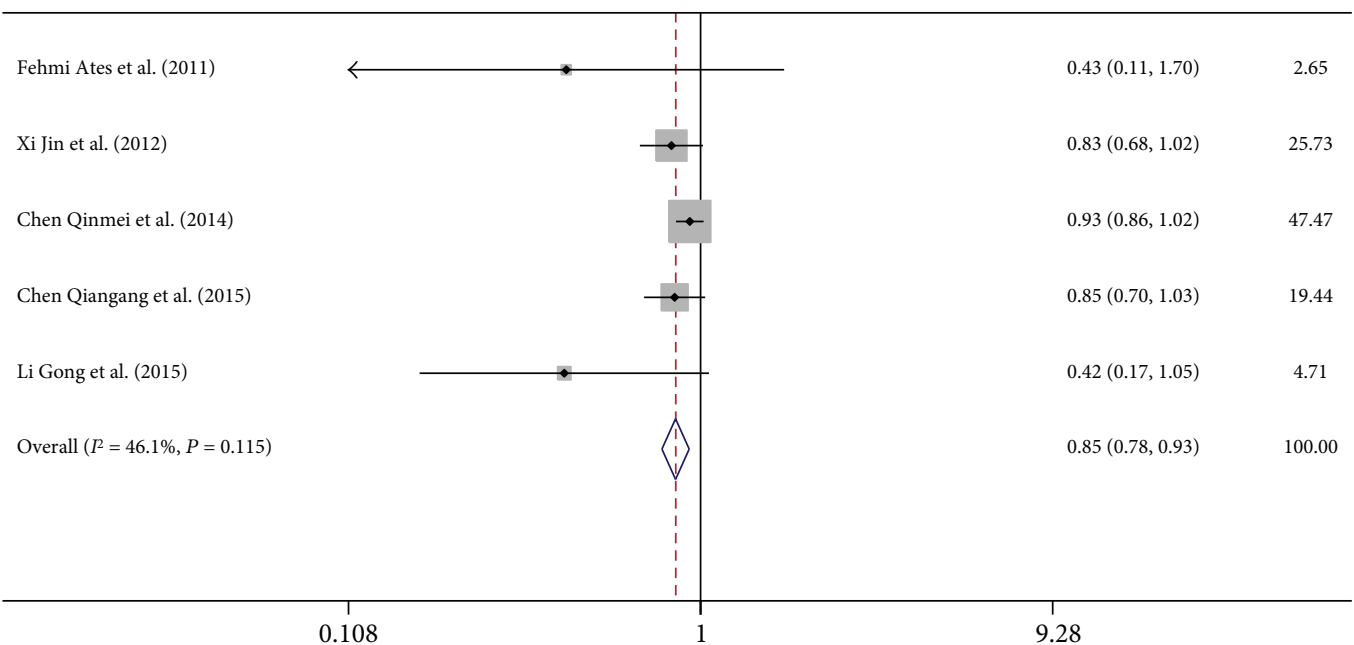

(b)

Figure 3: Continued. 


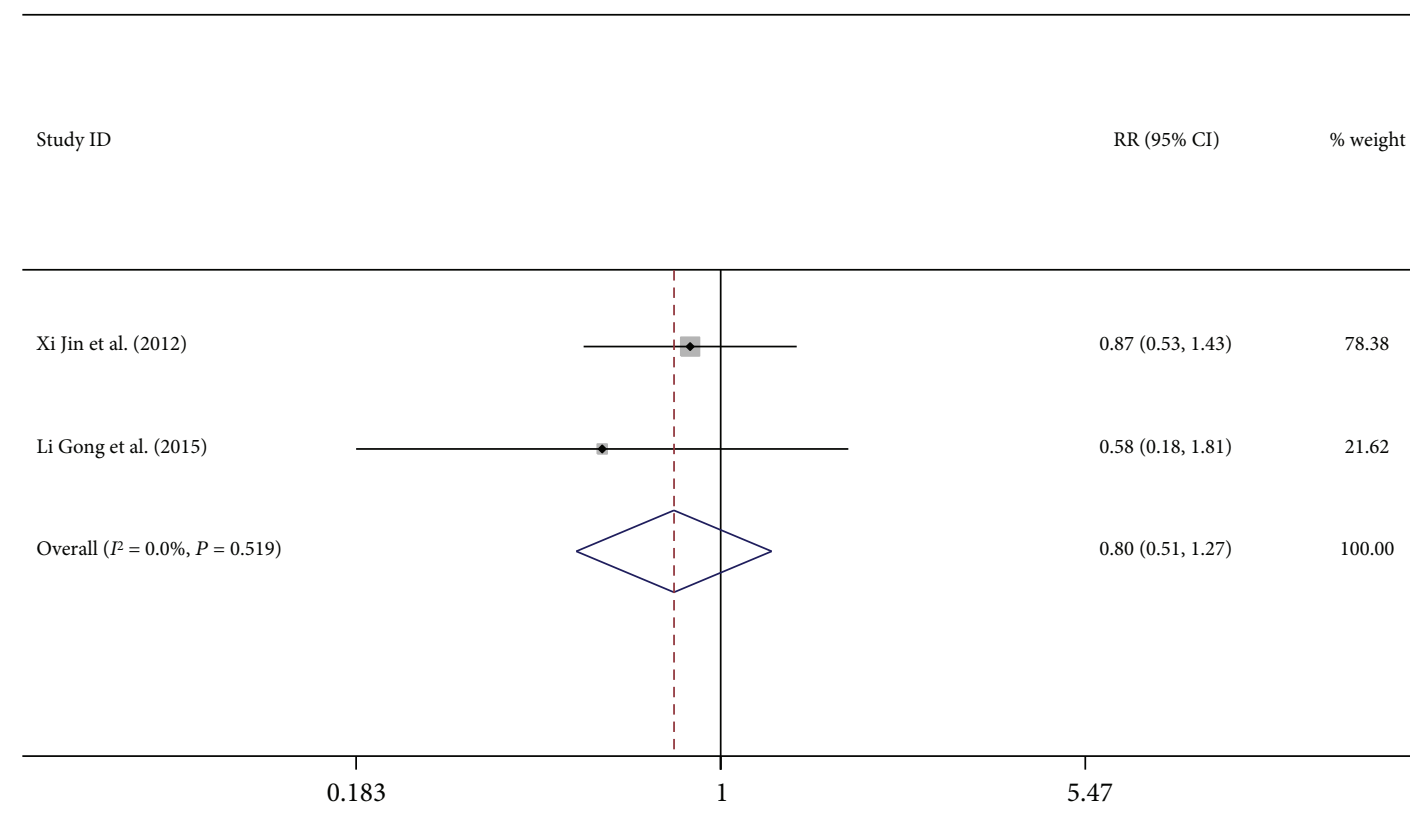

(c)

Figure 3: Forest plot of (a) biochemical, (b) virological, and (c) serological responses at 96 weeks.

TABLE 3: Subgroup analysis based on the method used for diagnosis of fatty liver.

\begin{tabular}{lcccc}
\hline Outcome or subgroup & Study & Participants & RR/WMD (weighted mean difference) 95\% CI & $P$ \\
\hline 48W biochemical response & 5 & $325 / 530$ & $0.864 /(0.778,0.960)$ & 0.007 \\
Histopathology & 2 & $65 / 120$ & $0.980 /(0.720,1.352)$ & 0.934 \\
Ultrasound B & 3 & $260 / 410$ & $0.842 /(0.754,0.940)$ & 0.002 \\
48W virological response & 7 & $380 / 623$ & $0.896 /(0.811,0.989)$ & 0.030 \\
Histopathology & 4 & $120 / 213$ & $1.002 /(0.773,1.298)$ & 0.987 \\
Ultrasound B & 3 & $260 / 410$ & $0.866 /(0.782,0.959)$ & 0.006 \\
48W serological response & 4 & $152 / 296$ & $0.900 /(0.661,1.226)$ & 0.504 \\
Histopathology & 3 & $87 / 148$ & $0.936 /(0.654,1.340)$ & 0.718 \\
Ultrasound B & 1 & $65 / 148$ & $0.828 /(0.458,1.498)$ & 0.533 \\
\hline
\end{tabular}

RR: risk ratio; CI: confidence interval.

$\mathrm{CHB}$ and hepatosteatosis started to receive antiviral treatment when their ALT levels and HBV DNA levels were abnormal according to the standard treatment criteria for $\mathrm{CHB}$ [27]. Moreover, all these studies applied Doppler ultrasound for the diagnosis of hepatosteatosis because of the abovementioned advantages. Why lower efficacy of antiviral therapy was observed in the patients with $\mathrm{CHB}$ and hepatosteatosis than in patients with $\mathrm{CHB}$ alone and the underlying mechanism remains unclear. Some researchers speculated that the ALT abnormity because of hepatosteatosis-induced liver chronic inflammatory was always misdiagnosed as the activation of $\mathrm{HBV}$, thus leading to early antiviral treatment, which may result in poor response to antiviral treatments in CHB patients with hepatosteatosis [18]. Our data were consistent with this speculation. This may be because experienced pathologists can distinguish $\mathrm{CHB}$ patients from CHB and NAFLD patients by biopsy, while doctors cannot differentiate CHB and NAFLD patients by Doppler ultrasound, which leads to the early antiviral treatment and thus poor response to treatments. Further studies are needed to confirm this since no detailed information for diagnosis was provided in the included studies. Anyway, our data suggest that, for $\mathrm{CHB}$ patients with hepatosteatosis, when the disease cause cannot be confirmed, biopsy can help us to confirm the cause and thus improve the response to antiviral treatments.

There are several limitations in this meta-analysis that ought to be highlighted. First, most studies included in the meta-analysis were retrospective, single-center studies. Secondly, the sample size in some of the studies was small. Both of these factors could have introduced an element of bias and affect the results of the meta-analysis. More prospective, multicenter observational studies are required to confirm our findings. 
Study ID

RR (95\% CI) \% weight

Histopathology

Shi Junping et al. (2012)

Li Gong et al. (2015)

Subtotal $\left(I^{2}=0.0 \%, P=0.925\right)$

Ultrasound B

Xi Jin et al. (2012)

Chen Qinmei et al. (2014)

Chen Qiangang et al. (2015)

Subtotal $\left(I^{2}=0.0 \%, P=0.850\right)$

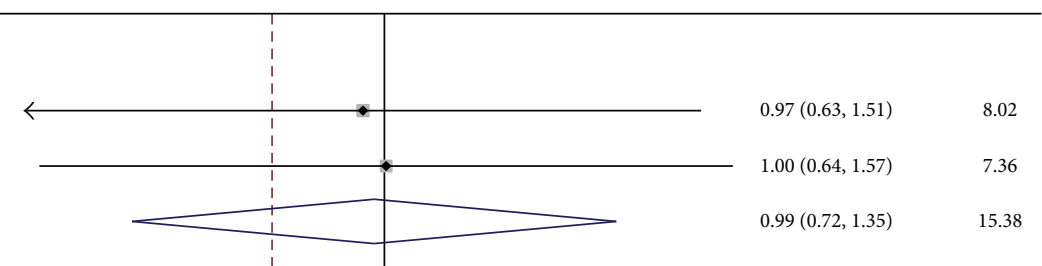

Overall $\left(I^{2}=0.0 \%, P=0.883\right)$

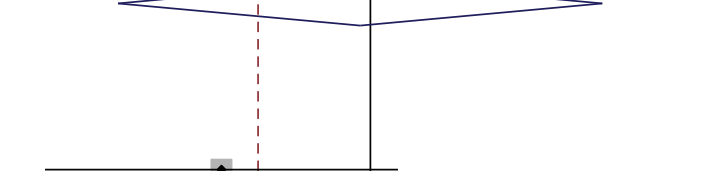

$0.82(0.66,1.04) \quad 24.19$

$0.87(0.75,1.00) \quad 42.80$

$0.81(0.63,1.03) \quad 17.63$

$0.84(0.75,0.94) \quad 84.62$

$0.86(0.78,0.96) \quad 100.00$

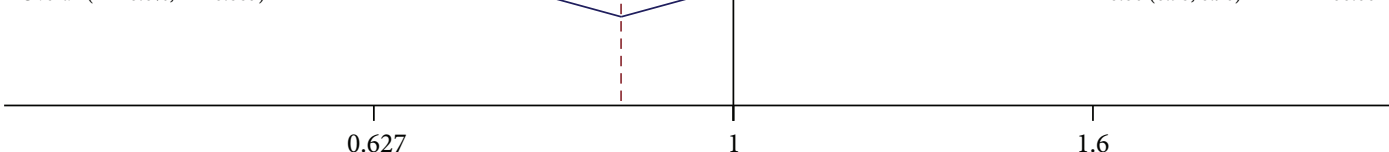

(a)

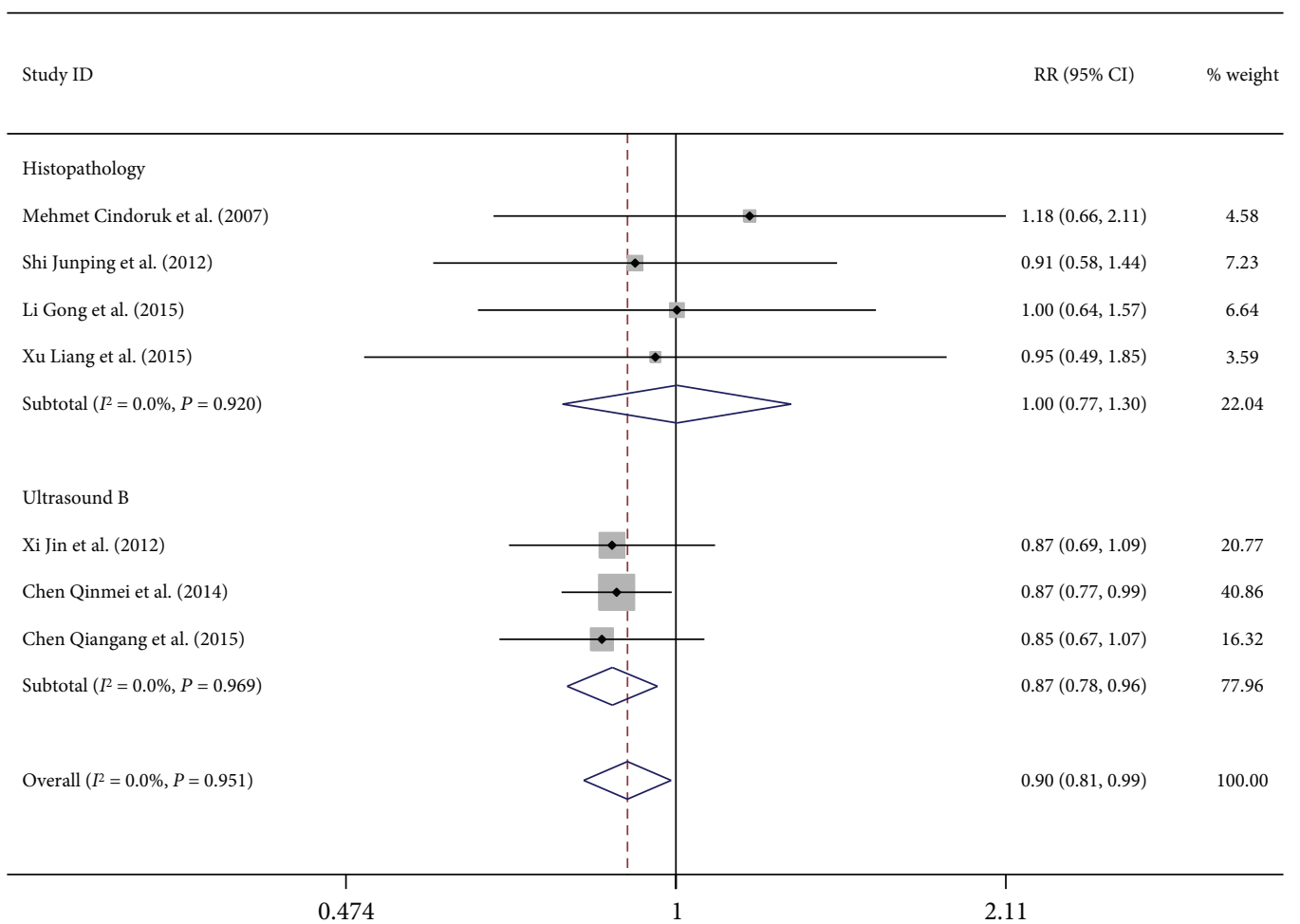

(b)

Figure 4: Continued. 


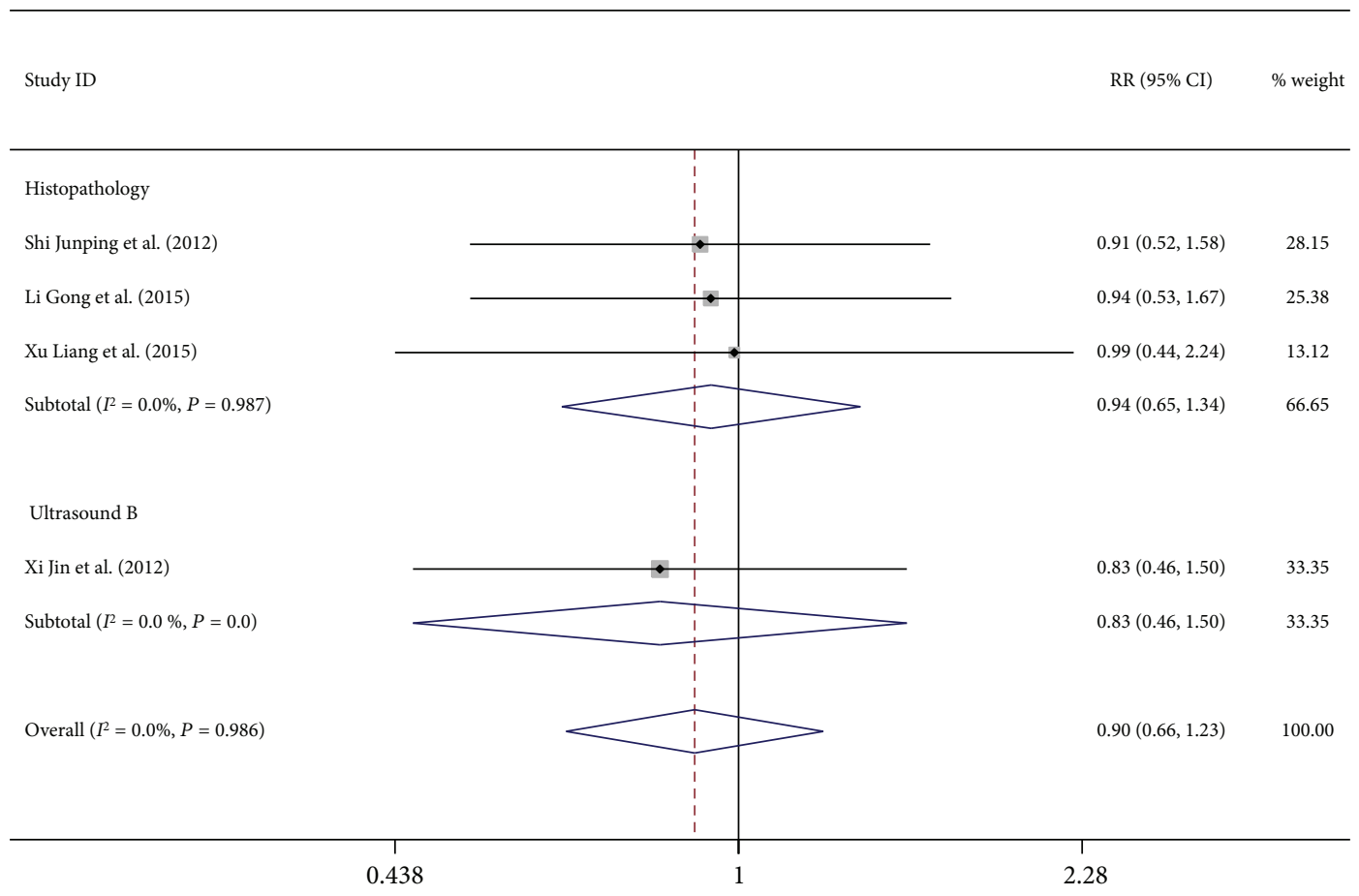

(c)

Figure 4: Subgroup analysis of (a) biochemical, (b) virological, and (c) serological responses at 48 weeks.

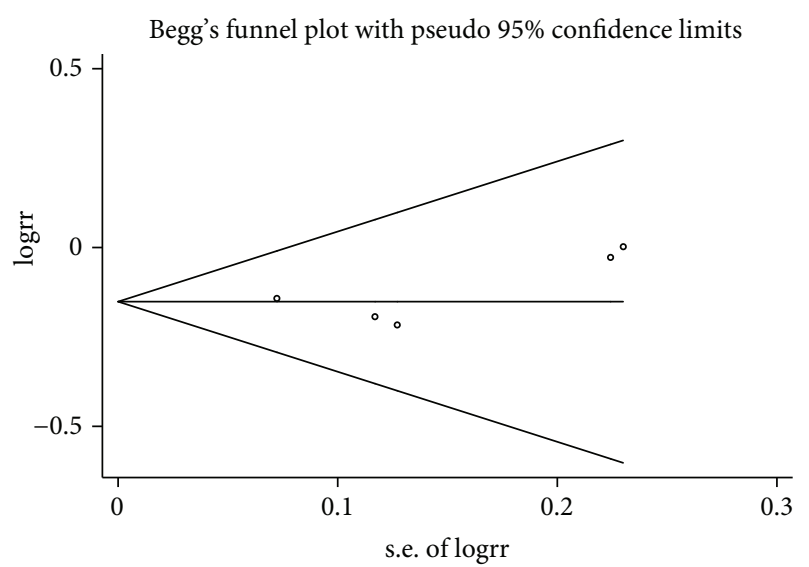

(a)

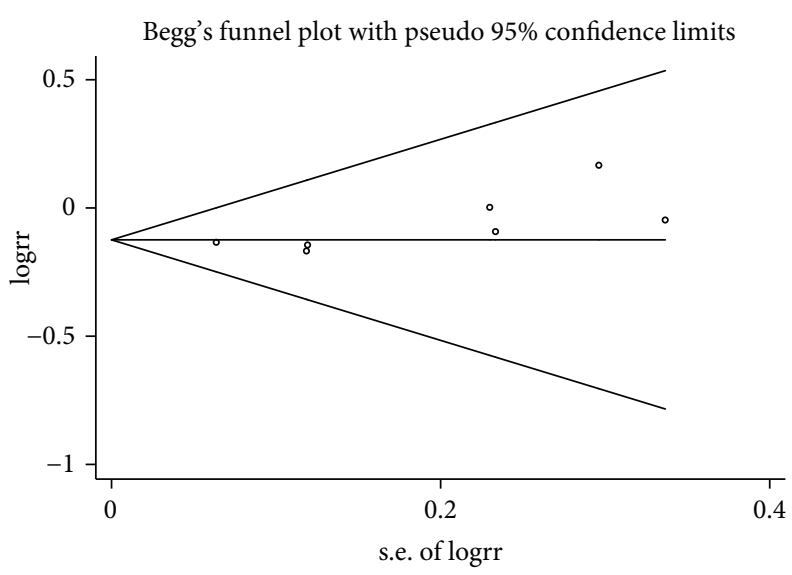

(b)

FIGURE 5: Begg's funnel plot of publication bias based on (a) biochemical and (b) virological responses at 48 weeks.

\section{Conclusion}

This meta-analysis indicates that hepatosteatosis in patients with $\mathrm{CHB}$ is associated with decreased response to antiviral treatment, especially when hepatosteatosis was diagnosed based on ultrasound findings and treated with nucleotide analogues.

\section{Conflicts of Interest}

The authors declare that they have no conflict of interest.

\section{Acknowledgments}

This study is supported by Zhejiang Provincial Natural Science Foundation of China (no. LY15H030009).

\section{References}

[1] G. Yonghao, X. Jin, L. Jun et al., “An epidemiological serosurvey of hepatitis $B$ virus shows evidence of declining prevalence due to hepatitis B vaccination in Central China," International Journal of Infectious Diseases, vol. 40, pp. 75-80, 2015. 
[2] M. F. Yuen and C. L. Lai, "Treatment of chronic hepatitis B: evolution over two decades," Journal of Gastroenterology and Hepatology, vol. 26, Supplement 1, pp. 138-143, 2011.

[3] N. Rosso, N. C. Chavez-Tapia, C. Tiribelli, and S. Bellentani, "Translational approaches: from fatty liver to non-alcoholic steatohepatitis," World Journal of Gastroenterology, vol. 20, no. 27, pp. 9038-9049, 2014.

[4] J. G. Fan, "Epidemiology of alcoholic and nonalcoholic fatty liver disease in China," Journal of Gastroenterology and Hepatology, vol. 28, no. Supplement 1, pp. 11-17, 2013.

[5] R. Loomba and A. J. Sanyal, "The global NAFLD epidemic," Nature Reviews Gastroenterology \& Hepatology, vol. 10, no. 11, pp. 686-690, 2013.

[6] R. Pais, E. Rusu, D. Zilisteanu et al., "Prevalence of steatosis and insulin resistance in patients with chronic hepatitis $B$ compared with chronic hepatitis $\mathrm{C}$ and non-alcoholic fatty liver disease," European Journal of Internal Medicine, vol. 26, no. 1, pp. 30-36, 2015.

[7] M. M. Wang, G. S. Wang, F. Shen, G. Y. Chen, Q. Pan, and J. G. Fan, "Hepatic steatosis is highly prevalent in hepatitis B patients and negatively associated with virological factors," Digestive Diseases and Sciences, vol. 59, no. 10, pp. 25712579, 2014.

[8] C. W. Lin, X. L. Huang, H. L. Liu, and Y. Wang, "Interactions of hepatitis B virus infection with nonalcoholic fatty liver disease: possible mechanisms and clinical impact," Digestive Diseases and Sciences, vol. 60, no. 12, pp. 3513-3524, 2015.

[9] Y. L. Wu, X. E. Peng, Y. B. Zhu, X. L. Yan, W. N. Chen, and $\mathrm{X}$. Lin, "Hepatitis B virus X protein induces hepatic steatosis by enhancing the expression of liver fatty acid binding protein," Journal of Virology, vol. 90, no. 4, pp. 1729-1740, 2016.

[10] S. Bondini, J. Kallman, A. Wheeler et al., "Impact of nonalcoholic fatty liver disease on chronic hepatitis B," Liver International, vol. 27, no. 5, pp. 607-611, 2007.

[11] C. C. Wang, T. C. Tseng, and J. H. Kao, "Hepatitis B virus infection and metabolic syndrome: fact or fiction?" Journal of Gastroenterology and Hepatology, vol. 30, no. 1, pp. 14-20, 2015.

[12] G. Wells, B. O. Shea, and D. Connell, The Newcastle-Ottawa Scale (NOS) for Assessing the Quality of Nonrandomized Studies in Meta-analyses, Ottawa (ON), Ottawa Health Research Institute, 2000.

[13] F. Ates, M. Yalniz, and S. Alan, "Impact of liver steatosis on response to pegylated interferon therapy in patients with chronic hepatitis B," World Journal of Gastroenterology, vol. 17, no. 40, pp. 4517-4522, 2011.

[14] M. Q. Chen, J. M. Wu, J. Chen, Y. Liu, Y. Xu, L. J. Wang et al., "The effect of non-alcoholic fatty liver disease on virologic response in patients with hepatitis $\mathrm{B}$ e antigen-positive chronic hepatitis B treated with nucleoside analogues," Chinese Journal of Infectious Diseases, vol. 32, 2014

[15] Q. G. Cheng, Y. J. Wang, X. Y. Ji, J. Feng, A. L. Li, L. Lin et al., "Efficacy of entecavir therapy for chronic hepatitis B patients; with non-alcoholic fatty liver disease," Chinese Journal of Clinical Infectious Diseases, vol. 8, 2015.

[16] M. Cindoruk, T. Karakan, and S. Unal, "Hepatic steatosis has no impact on the outcome of treatment in patients with chronic hepatitis B infection," Journal of Clinical Gastroenterology, vol. 41, no. 5, pp. 513-517, 2007.

[17] L. Gong, J. Liu, J. Wang, G. Q. Lou, and J. P. Shi, "Hepatic steatosis as a predictive factor of antiviral effect of pegylated interferon therapy in patients with hepatitis B," Transplantation Proceedings, vol. 47, no. 10, pp. 2886-2891, 2015, Epub 2015/12/29.

[18] X. Jin, Y. P. Chen, Y. D. Yang, Y. M. Li, L. Zheng, and C. Q. Xu, "Association between hepatic steatosis and entecavir treatment failure in Chinese patients with chronic hepatitis B," PloS One, vol. 7, no. 3, article e34198, 2012.

[19] J. P. Shi, L. Lu, J. C. Qian, J. Ang, Y. H. Xun, J. C. Guo et al., "Impact of liver steatosis on antiviral effects of pegylated interferon-alpha in patients with chronic hepatitis B," Zhonghua Gan Zang Bing Za Zhi, vol. 20, no. 4, pp. 285-288, 2012.

[20] L. Xu, P. Li, Q. Shi, and Y. Mi, "Impact of liver steatosis on the curative effect of pegylated interferon-alpha-2a in patients with chronic hepatitis B," Zhonghua Gan Zang Bing Za Zhi, vol. 23, no. 2, pp. 99-102, 2015.

[21] M. Sunbul, "Hepatitis B virus genotypes: global distribution and clinical importance," World Journal of Gastroenterology, vol. 20, no. 18, pp. 5427-5434, 2014.

[22] R. Zhou, Y. P. Zhou, C. Lin et al., "Baseline prognostic factors and statistic model to predict early virological response in telbivudine-treated patients with chronic hepatitis B," Hepatitis Monthly, vol. 13, no. 12, article e15573, 2013.

[23] L. B. Yan, E. Q. Chen, L. Bai et al., "Efficacy of entecavir treatment for up to 96 weeks in nucleoside-naive HBeAgpositive chronic hepatitis B patients with high viral load," Clinics and Research in Hepatology and Gastroenterology, vol. 39, no. 3, pp. 366-372, 2015.

[24] P. Bedossa and T. Poynard, "An algorithm for the grading of activity in chronic hepatitis C. The METAVIR cooperative study group," Hepatology, vol. 24, no. 2, pp. 289-293, 1996.

[25] D. E. Kleiner, E. M. Brunt, M. Van Natta et al., "Design and validation of a histological scoring system for nonalcoholic fatty liver disease," Hepatology, vol. 41, no. 6, pp. 1313-1321, 2005.

[26] E. Hashimoto, M. Taniai, and K. Tokushige, "Characteristics and diagnosis of NAFLD/NASH," Journal of Gastroenterology and Hepatology, vol. 28, Supplement 4, pp. 64-70, 2013.

[27] Chinese Society of H, Chinese Society of Infectious Diseases CMA, "The guideline of prevention and treatment for chronic hepatitis B (2010 version)," Zhonghua Liu Xing Bing Xue Za Zhi, vol. 32, no. 4, pp. 405-415, 2011. 


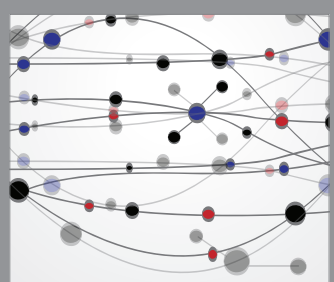

The Scientific World Journal
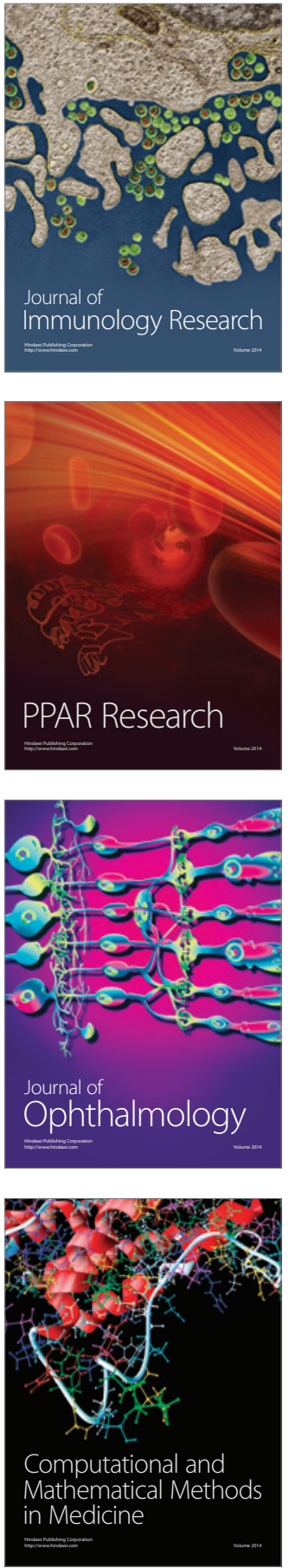

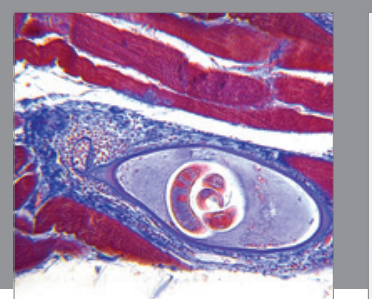

Gastroenterology Research and Practice
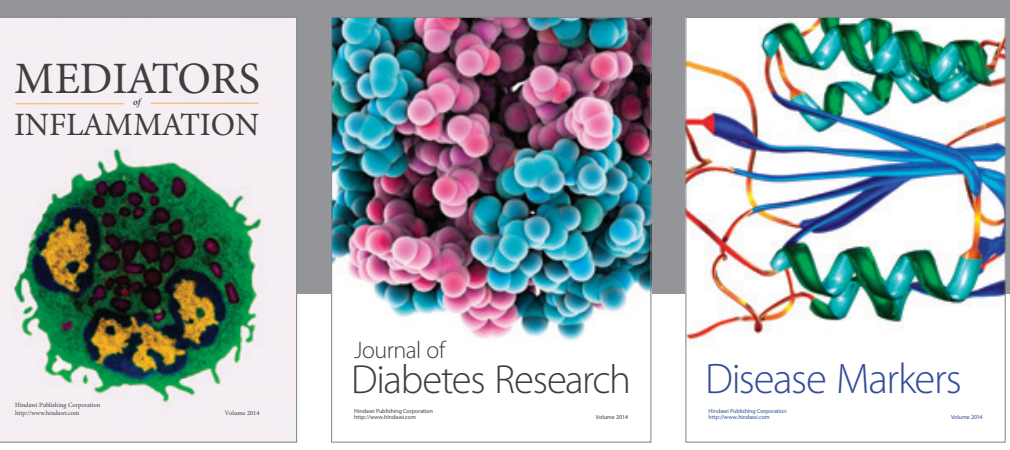

Disease Markers

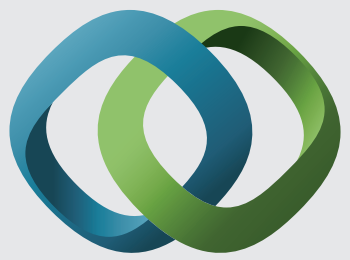

\section{Hindawi}

Submit your manuscripts at

https://www.hindawi.com
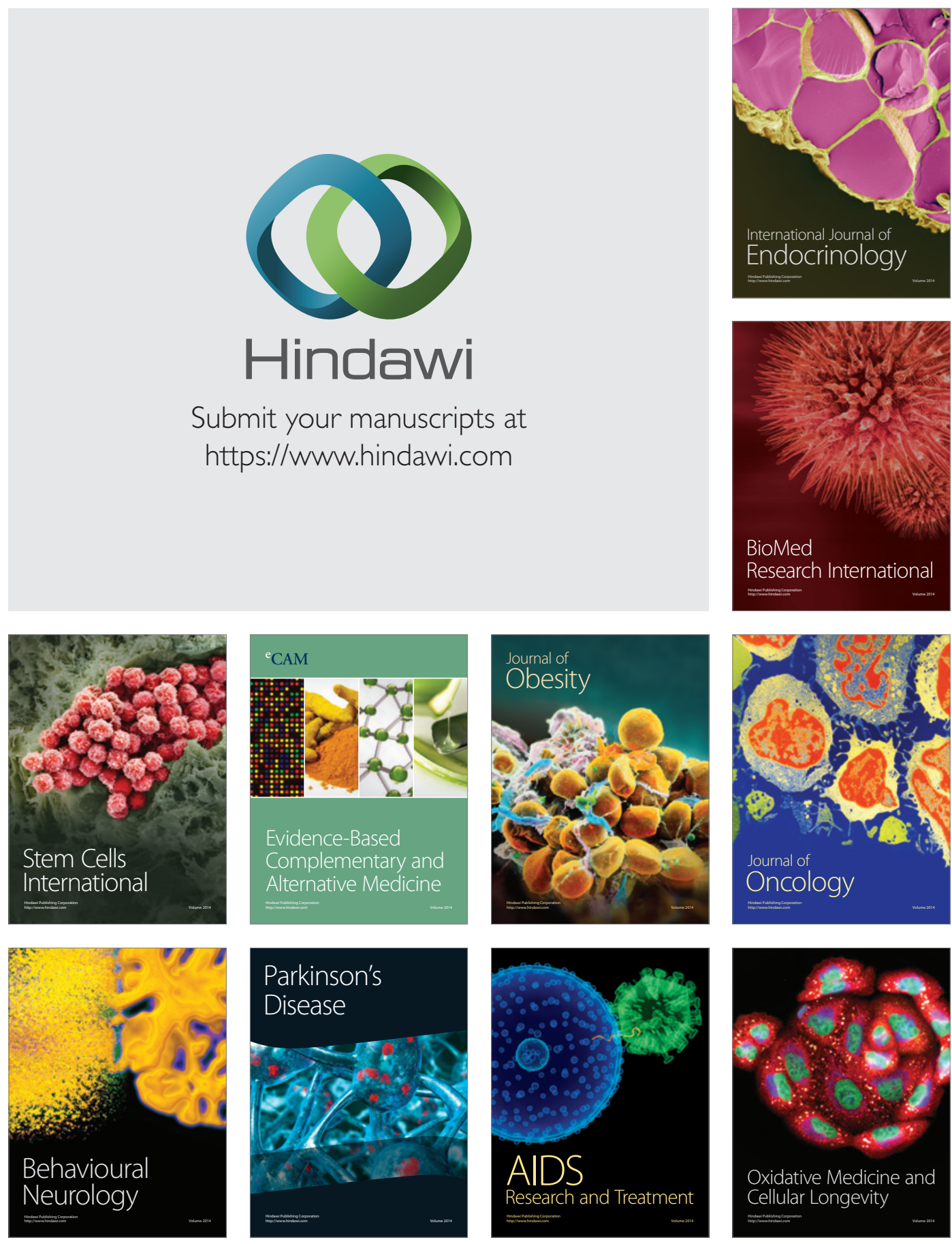